sible forms of $J(t)$. For example, we cannot obtain the response to an impulse function and cannot even hope to approximate to it reasonably by an f.f.t. of economical size. The same type of comment applies, although less forcibly, for a step-function input. These are the penalties for having an incomplete set of data, and cannot be avoided. We can, however, obtain the response to practical inputs in which the risetime is finite, even though the process may be expensive if the risetime is small. Again, we must emphasise that this is the only known solution to this type of problem in which we require the solution of a differential equation we cannot form.

For a nonlinear circuit, we assume that the nonlinearities are in the lumped portion of the circuit and are of the type that may be represented by voltage-dependent current generators. If we knew the differential operator $y(t)$ we could write down a set of differential equations describing the system

$$
y(t) v(t)=J(t)+f[v(t)]
$$

where $f$ is a vector of known nonlinear functions. The solution of eqn. 2 is given by the Volterra-integral equation

$$
v(t)=v^{(0)}(t)+\int_{0}^{t} H(t-\tau) f[v(\tau)] d \tau
$$

As before, $y(t)$ is not explicitly defined; all we know are the values of $\boldsymbol{Y}(\omega)$ at specific frequencies. $\boldsymbol{v}^{(0)}(t)$ is obtained, as in the linear case, by finding the i.f.f.t. of $Z(\omega) I(\omega)$, and $\boldsymbol{H}(t)$ is the inverse Fourier transform of $\boldsymbol{Z}(\omega)$, and is therefore defined at discrete points. Subject to convergence, $v(t)$ may then be found by a Picard iteration, forming successively $\boldsymbol{v}^{(0)}, \boldsymbol{v}^{(1)}, \boldsymbol{v}^{(2)}, \ldots$, using the recurrence relation

$$
\boldsymbol{v}^{(j+1)}(t)=\boldsymbol{v}^{(0)}(t)+\int_{0}^{t} \boldsymbol{H}(t-\tau) \boldsymbol{f}\left[\boldsymbol{v}^{(j)}(\tau)\right] d \tau . .
$$

where, at each stage, the convolution integral is evaluated by forming the f.f.t. $\boldsymbol{F}(\omega)$ of $f\left[\boldsymbol{v}^{(j)}(t)\right]$ and, for each $\omega$, premultiplying this vector by the corresponding $Z(\omega)$ and, finally, retransforming, via the i.f.f.t., into the time domain. This type of iteration has, in recent years, received detailed consideration by this writer and others ${ }^{1-4}$ in its application to finding the steady-state response of a nearly linear system. Present considerations show that the desirable applications of eqn. 3 are wider than were originally thought, since, for a wide class of circuits, it forms the only known method of analysis. However, in addition to the difficulties that have already been observed in the linear case, there are now additional difficulties arising from the limited region of convergence of the Picard iteration. It is shown ${ }^{4}$ that, for reasonably well behaved functions $f$, convergence of the iteration may be obtained in otherwise nonconvergent cases by the introduction of a damping matrix $C$, modifying eqn. 3 to

$$
v(t)=C v^{(0)}(t)+(U-C) v(t)+C \int_{0}^{t} H(t-\tau) f[v(\tau)] d \tau
$$

where $U$ is the unit matrix, and, using the iteration,

$$
\begin{aligned}
v^{(1)}(t) & =(2 U-C) C v^{(0)}+C \int_{0}^{t} H(t-\tau) f\left[C v^{(0)}(\tau)\right] d \tau \\
v^{(j+1)}(t) & =C v^{(0)}(t)+(U-C) v^{(j)}(t) \\
& +C \int_{0}^{t} H(t-\tau) f\left[v^{(j)}(\tau)\right] d \tau \quad j \neq 0
\end{aligned}
$$

In principle, again for reasonably well behaved $f$, it is always possible to find a matrix $C$ such that this iteration converges, but, for grossly nonlinear circuits, this result is generally not a useful one as the progress towards a solution may be so slow as to be uneconomical, even if it is not completely lost in the rounding errors of the computer. Higher-order iterative solutions such as the Newton-Raphson iteration also become impracticably unwieldy for any but the simplest systems.
Thus the present situation may be summarised by saying that, with the techniques currently available, nonlinear circuits that cannot be modelled in terms of lumped elements can be analysed in the manner described provided that the nonlinearities are not too severe. No other method for obtaining such results seems to be available. No method is known by which a solution can be obtained economically in grossly nonlinear cases, and further work is required in this respect. By using the generalisation of nodal analysis described in an earlier letter, ${ }^{5}$ all four types of dependent generator may be accommodated in this type of analysis.

T. B. M. NEILL

18th February 1974

\section{Post Office Research Department}

Dollis Hill

London NW2 7DT, England

\section{References}

1 NEILL, T. B. M.: 'Improved method of analysing nonlinear electrical networks', Electron. Lett., 1969, 5, pp. 13-15

2 HEYWOOD, D. R., and MOORE, A. D.: 'Comment on an improved method of analysing nonlinear electrical networks', ibid., 1969, 5 pp. $269-270$

3 NEILL, T. B. M.: 'Reply to comment on an improved method of analysing nonlinear electrical networks', ibid, 1969, 5, pp. 270-27

4 NEILL, T. B. M. 'Spectral analysis of nonlinear circuits' in 'Network and signal theory'. PPL Conf. Publ. 12, 1972, pp. 122-131

5 NEILL, T. B. M.: 'Generalisation of nodal and mesh analysis', Electron. Lett., 1969, 5, pp. 365-366

\section{SELECTION OF ZEROS OF REFLECTION COEFFICIENT IN DESIGN OF FILTERS BY INSERTION LOSS}

\begin{abstract}
Indexing terms: Filters, Network synthesis, Poles and zeros
It is shown that a characteristic function that can be expressed as the square of an odd rational function in $\omega$, with poles on the $j \omega$ axis and at infinity, is always realisable without using transformers by the selection of the zeros of $p(s)$, and this is the key for a filter design based on 2-port synthesis theory.
\end{abstract}

If one wants to insert an $L C$ filter between a unity-internalresistance generator and a load resistance $r$ (both normalised), its transmission characteristic may be expressed by means of its insertion-loss function $P_{20} / P_{2}$, or its characteristic function $F\left(\omega^{2}\right)$, related by

$$
\frac{P_{20}}{P_{2}}=1+F\left(\omega^{2}\right)
$$

Defining

$$
\rho(s) \rho(-s)=1-\left.t \frac{P_{2}}{P_{20}}\right|_{\omega^{2}=-s^{2}}
$$

with $t=4 r /(1+r)^{2}$; the filter input immittance is ${ }^{1}$

$$
Z(s) \text { or } Y(s)=\frac{1-\rho(s)}{1+\rho(s)}=\frac{m_{1}+n_{1}}{m_{2}+n_{2}}
$$

and its transfer function is

$$
H(s)=\frac{E_{2}}{E_{20}}=\frac{1+r}{r} \frac{\sqrt{ }\left(m_{1} m_{2}-n_{1} n_{2}\right)}{m_{1}+m_{2}+n_{1}+n_{2}} . .
$$

For a lowpass filter-other band configurations are obtained by a frequency transformation-the following expressions ${ }^{2}$ give its parameter matrix:

$$
\begin{aligned}
& Z_{11} \text { or } Y_{11}=\frac{m_{1}}{n_{2}} \\
& \frac{1}{\sqrt{ } r} Z_{21} \text { or }-\sqrt{ } r Y_{21}=\frac{\sqrt{ }\left(m_{1} m_{2}-n_{1} n_{2}\right)}{n_{2}} \\
& \frac{1}{r} Z_{22} \text { or } r Y_{22}=\frac{m_{2}}{n_{2}}
\end{aligned}
$$


If $F\left(\omega^{2}\right)>0$ for all $\omega$, and its poles are located on the $j \omega$ axis, taking from eqn. 1 the left-halfplane poles of $\rho(s)$, eqn. 2 gives an immittance that is a real positive function, and the parameters from eqn. 4 will constitute an odd real positive matrix, which leads to a practical ladder filter without transformers, provided that the Fialcow-Gerst ${ }^{3}$ conditions are met. At this point, the selection of the zeros of $\rho(s)$ is of greatest importance.

Let $N$ be an odd and $M$ an even polynomial in $s$ satisfying

(a) degree of $N>$ degree of $M$

(b) highest-degree-term coefficient of $N$ equals 1 .

Taking

$$
F\left(\omega^{2}\right)=-\left.\frac{N^{2}}{M^{2}}\right|_{s=j \omega}
$$

as the characteristic function, ${ }^{*}$ then

$$
\frac{P_{2}}{P_{20}}=\left.H(s) H(-s)\right|_{s=j \omega}=\left.\frac{M^{2}}{M^{2}-N^{2}}\right|_{s=j \omega}
$$

and, from eqns. 3 and 1 ,

$$
\sqrt{ }\left(m_{1} m_{2}-n_{1} n_{2}\right)=\frac{\sqrt{ } r}{1+r} M
$$

and

$$
\rho(s) \rho(-s)=\frac{\left(m_{2}-m_{1}\right)^{2}-\left(n_{2}-n_{1}\right)^{2}}{\left(m_{2}+m_{1}\right)^{2}-\left(n_{2}+n_{1}\right)^{2}}=\frac{(1-t) M^{2}-N^{2}}{M^{2}-N^{2}}
$$

Obviously, from eqn. 6 , the immittance has a zero or a pole at infinity; hereafter it will be supposed to have a zero, without loss of generality, and

$$
\rho(0)= \pm \frac{1-r}{1+r} \text {. }
$$

To form $\rho(s)$, the left-halfplane roots of $M^{2}-N^{2}$ must be obtained, giving $M_{1}+N_{1}$. It may be easily proved that

(a) degree of $N_{1}=$ degree of $N$

(b) degree of $N_{1}>$ degree of $M_{1}$

(c) $M_{1}(0)=M(0)$

(d) $N_{1}$ highest-degree-term coefficient equals 1

(e) the coefficient of a given power of $s$ in $M_{1} \geqslant$ the corresponding coefficient in $M$ (coefficients $M_{1} \geqslant$ coefficients $M)$.

The selection of the zeros of $\rho(s)$ that guarantees the fulfilment of the Fialcow-Gerst conditions by the matrix of eqn. 4 leads to

$$
\rho(s)=\frac{\rho(0) M+N}{M_{1}+N_{1}}
$$

where $\rho(0)$ is given in eqn. 7 , and, reserving the symbol + to obtain $Z(s)$ and the symbol - for $Y(s)$, is equivalent to taking $Z(0)=r$ and $Y(0)=1 / r$.

From eqns. 2, 4 and 5, the proof that this choice of $\rho(s)$ is correct reduces to verifying that

coefficients $\frac{1}{2}\left(M_{1}-\frac{1-r}{1+r} M\right) \geqslant$ coefficients $\left.\frac{r}{1+r} M\right)$

and

coefficients $\frac{r}{2}\left(M_{1}+\frac{1-r}{1+r} M\right) \geqslant$ coefficients $\left.\frac{r}{1+r} M\right)$

where the factor $1 / 2$ has been introduced, so that

$$
n_{2}=1 / 2\left(N_{1}+N\right)
$$

has its highest-degree coefficient equal to unity, and to preserve consistency with eqn. 6 ; verification of eqn. 8 is immediate once it has been established that coefficients $M_{1} \geqslant$ coefficients $M$.

* Obviously, we suppose the zeros of $M$ to be on the $j \omega$ axis
It is interesting to mention that, when $M$ is a constant, all filter transmission zeros at infinity, since the zero-degree numerator term is independent of the choice of the zeros of $\rho(s)$, the fulfilment of the Fialcow-Gerst conditions will not depend on that selection.

J. B. MARIÑO

22nd February 1974

ETS Ingenieros de Telecomunicación

Universidad Politécnica de Barcelona

331 Avenida del Caudillo

Barcelona, Spain

\section{References}

1 TUTTLE, D. F.: 'Redes eléctricas' (Dossat, Madrid, 1964), chap. 13

2 VLACH, J.: "Computerized approximation and synthesis of linear networks' (Wiley, 1969), chap. 4

3 FIALCOW, A. D., and GERST, l.: 'The transfer function of general two terminal-pair $R C$ - networks', Quart. Appl. Math., 1952, 10, pp. 113-127

\section{OBLIQUITY FACTOR FOR RADIATION FROM SOLID-STATE LASER}

Indexing terms: Laser beams, Solid lasers

The radiated field consists of forward radiation at angle $\theta$ and backward radiation at $\pi-\theta$ reflected from the dielectricair interface into an additional component of field at angle $\theta$. The total radiation contains a factor that simplifies into an The total radiation contains a factor that simplifies into an and which is here interpreted as the Huygens obliquity factor for the arrangement. A small correction to the expression is also obtained.

Recently, ${ }^{1}$ by means of mathematical techniques involving Fourier integrals and saddle-point asymptotic evaluations, expressions have been produced for some properties of solidstate-laser radiation, including the damped interference waves at the dielectric-air boundary, and an expression leading to the observed sharpening of the radiation-pattern. The latter factor can be written

$\left.\left.g(\theta)=\frac{2 \cos \theta}{\left\{\cos \theta+\sqrt{ }\left(\varepsilon_{2}-\sin ^{2} \theta\right)\right.}\right\} \beta / k_{0}+\sqrt{ }\left(\varepsilon_{2}-\sin ^{2} \theta\right)\right\}$

where

$$
\begin{aligned}
g(\theta)= & \text { radiation-pattern factor additional to main } \\
& \text { radiation-pattern expression } \\
\theta= & \text { angle off-axis at which the field is observed } \\
\varepsilon_{2}= & \text { relative permittivity of laser material } \\
\beta= & \text { axial propagation factor in laser } \\
k_{0}= & \text { propagation factor in air }
\end{aligned}
$$

It is interesting to note that eqn. 1 can be obtained by elementary means, and that it represents, for the dielectric-air configuration, the form taken by the Huygens obliquity factor.*

Fig. 1 shows the arrangement, with an incident surface wave of the form ${ }^{1}$

$$
E_{y}=E_{l}(x) \exp (-j \beta z) \quad z<0 .
$$

where $E_{l}(x)$ is the transverse-field distribution of the incident surface-wave mode. In region 3 (air), the field can be found from the form of Huygens's integral in cylindrical co-ordinates: ${ }^{2}$

$$
\begin{aligned}
& \psi=\left(8 \pi k_{0}\right)^{-\frac{1}{2}} \exp (-j \pi / 4) \int_{-\infty}^{\infty}\left\{\frac{\partial \psi_{0}}{\partial n} \frac{\exp \left(-j k_{0} r\right)}{\sqrt{ } r}\right. \\
&\left.-\psi_{0} \frac{\partial}{\partial n} \frac{\exp \left(-j k_{0} r\right)}{\sqrt{ } r}\right\} d x
\end{aligned}
$$

* The Huygens obliquity factor is the radiation pattern for an element of a radiating aperture. It has the value $\cos \phi+\cos \theta$ for radiation at an angle $\theta$ for
incident radiation at an angle $\phi$. It is slowly varying, and is often ignored when incident radiation at an angle $\phi$. It is slowly varying,
calculating radiation patterns from large apertures 